New results on the AM-GM inequality

\author{
Božidar Ivanković, Josip Pečarić, and Sanja \\ Varošanec
}




\title{
NEW RESULTS ON THE AM-GM INEQUALITY
}

\author{
BOŽIDAR IVANKOVIĆ, JOSIP PEČARIĆ, AND SANJA VAROŠANEC \\ Received 11 May, 2010
}

\begin{abstract}
There is a large number of discrete inequalities between geometric and arithmetic means. Our intention is to investigate possible generalization and integral analogues of the inequalities obtained by Redhefer, Bullen and Godunova. At the end, we will consider refinements of these inequalities by different separations of their domains.
\end{abstract}

2000 Mathematics Subject Classification: 26D15

Keywords: convex functions, Jensen's inequality

\section{Preliminares}

Consider the following sequences of positive real numbers:

$(a)=\left(a_{1}, \ldots, a_{n}\right) ;(b)=\left(b_{1}, \ldots, b_{n}\right) ;(p)=\left(p_{1}, \ldots, p_{n}\right) ;(q)=\left(q_{1}, \ldots, q_{n}\right)$.

We use the notations:

$$
\begin{gathered}
P_{n}=\sum_{k=1}^{n} p_{k}, Q_{n}=\sum_{k=1}^{n} q_{k}, \\
A_{n}(a, q)=\frac{1}{Q_{n}} \sum_{k=1}^{n} q_{k} a_{k}, G_{n}(a, q)=\prod_{k=1}^{n} a_{k}^{\frac{q_{k}}{Q_{n}}}, \\
\Gamma_{n}(a, q)=\frac{1}{Q_{n}} \sum_{k=1}^{n} q_{k} G_{k}(a, q) .
\end{gathered}
$$

A function $F:[a, b] \rightarrow \mathbb{R}$ is said to be convex if for all $x, y \in[a, b]$ and all $\alpha \in$ $[0,1]$,

$$
F(\alpha x+(1-\alpha) y) \leq \alpha F(x)+(1-\alpha) F(y) .
$$

If the inequality (1.2) is reversed, then $F$ is said to be concave.

Investigating recurrent inequalities in [5], Redhefer has obtained the following result involving the arithmetic and geometric means. The statement is given in terms of $(1.1)$, where $G_{n}(a, 1)=G_{n}(a, q), q_{k}=1, k=1, \ldots, n$. 
Theorem 1 (Redhefer). If $t>0$, then

$$
G_{n}(a, 1)<A_{n}(a, 1) e^{-t}+t \Gamma_{n}(a, 1) .
$$

Bullen [2] proved an inequality of Rado type generalizing Redheffer's inequality.

Theorem 2 (Bullen). If $0 \leq t q_{n} \leq Q_{n}$, then

$$
\begin{array}{r}
Q_{n}\left(A_{n}(a, q) e^{-t}+t \Gamma_{n}(a, q)-G_{n}(a, q)\right) \\
\geq Q_{n-1}\left(A_{n-1}(a, q) e^{-t}+t \Gamma_{n-1}(a, q)-G_{n-1}(a, q)\right) .
\end{array}
$$

A more general result (given bellow) is obtained in [3].

Theorem 3 (Godunova). Suppose that $G$ and $F$ are twice continuously differentiable functions such that $G$ is concave and $G F=(G \circ F)$ is convex.

For $t \in \mathbb{R}$ the next inequality is true:

$$
\begin{array}{r}
Q_{n}\left\{G\left(A_{n}\left(F\left(t+a_{k}\right), q\right)\right)-t A_{n}\left((G F)^{\prime}\left(\frac{q_{k} P_{k}}{p_{k} Q_{k}} A_{k}(a, p)\right), q\right)\right. \\
\left.-G F\left(\frac{q_{n} P_{n}}{p_{n} Q_{n}} A_{n}(a, p)\right)\right\} \\
\geq Q_{n-1}\left\{G\left(A_{n-1}\left(F\left(t+a_{k}\right), q\right)\right)-t A_{n-1}\left((G F)^{\prime}\left(\frac{q_{k} P_{k}}{p_{k} Q_{k}} A_{k}(a, p)\right), q\right)\right. \\
\left.-G F\left(\frac{q_{n} P_{n-1}}{p_{n} Q_{n-1}} A_{n-1}(a, p)\right)\right\} .
\end{array}
$$

In this article we will present some generalizations and refinements of these results.

\section{MAIN RESUlT}

Our results are based on the well-known properties of convex functions. The conditions are simpler than the ones of Theorem 3. It is obvious that Theorem 3 follows from this result.

Theorem 4. If $F$ is a convex differentiable function, then for all $t \in \mathbb{R}$

$$
\begin{array}{r}
Q_{n}\left[A_{n}\left(F\left(t+a_{k}\right), q\right)-t \cdot A_{n}\left(F^{\prime}\left(\frac{q_{k} P_{k}}{p_{k} Q_{k}} \cdot A_{k}(a, p)\right), q\right)\right. \\
\left.-F\left(\frac{q_{n} P_{n}}{p_{n} Q_{n}} \cdot A_{n}(a, p)\right)\right] \\
\geq Q_{n-1}\left[A_{n-1}\left(F\left(t+a_{k}\right), q\right)-t \cdot A_{n-1}\left(F^{\prime}\left(\frac{q_{k} P_{k}}{p_{k} Q_{k}} \cdot A_{k}(a, p)\right), q\right)\right. \\
\left.-F\left(\frac{q_{n} P_{n-1}}{p_{n} Q_{n-1}} \cdot A_{n-1}(a, p)\right)\right] .
\end{array}
$$


Proof. Obviously, we have

$$
\sum_{k=1}^{n} q_{k} F\left(t+a_{k}\right)-\sum_{k=1}^{n-1} q_{k} F\left(t+a_{k}\right)=q_{n} F\left(t+a_{n}\right)
$$

and

$$
\begin{aligned}
Q_{n} \cdot A_{n}\left(F^{\prime}\left(\frac{q_{k} P_{k}}{p_{k} Q_{k}} \cdot A_{k}(a, p)\right), q\right) & \\
-Q_{n-1} \operatorname{cdot} A_{n-1}\left(F ^ { \prime } \left(\frac{q_{k} P_{k}}{p_{k} Q_{k}}\right.\right. & \left.\left.\cdot A_{k}(a, p)\right), q\right) \\
& =q_{n} \cdot F^{\prime}\left(\frac{q_{n} P_{n}}{p_{n} Q_{n}} \cdot A_{n}(a, p)\right) .
\end{aligned}
$$

Statement (2.1) is equivalent to

$$
\begin{array}{r}
q_{n} F\left(t+a_{n}\right)+Q_{n-1} \cdot F\left(\frac{q_{n} P_{n-1}}{p_{n} Q_{n-1}} \cdot A_{n-1}(a, p)\right) \\
\geq Q_{n} F\left(\frac{q_{n} P_{n}}{p_{n} Q_{n}} \cdot A_{n}(a, p)\right)+t q_{n} F^{\prime}\left(\frac{q_{n} P_{n}}{p_{n} Q_{n}} \cdot A_{n}(a, p)\right),
\end{array}
$$

so it remains to show (2.2). Because of $\frac{q_{n}}{Q_{n}}+\frac{Q_{n-1}}{Q_{n}}=1$, the definition of convex functions implies

$$
\begin{aligned}
q_{n} \cdot F\left(t+a_{n}\right) & +Q_{n-1} \cdot F\left(\frac{q_{n} P_{n-1}}{p_{n} Q_{n-1}} \cdot A_{n-1}(a, p)\right) \\
& \geq Q_{n} \cdot F\left(\frac{t q_{n}}{Q_{n}}+\frac{q_{n} P_{n}}{p_{n} Q_{n}} \cdot A_{n}(a, p)\right) .
\end{aligned}
$$

Presuming that the convex function $F$ is differentiable, we have (see [4], p.5, Theorem 1.6.):

$$
F(u+\alpha) \geq F(u)+\alpha \cdot F^{\prime}(u) .
$$

Upon application of these results, the following inequality is obtained

$$
\begin{aligned}
& Q_{n} \cdot F\left(\frac{q_{n} P_{n}}{p_{n} Q_{n}} \cdot A_{n}(a, p)+\frac{t q_{n}}{Q_{n}}\right) \\
& \geq Q_{n} \cdot F\left(\frac{q_{n} P_{n}}{p_{n} Q_{n}} \cdot A_{n}(a, p)\right)+t \cdot q_{n} \cdot F^{\prime}\left(\frac{q_{n} P_{n}}{p_{n} Q_{n}} \cdot A_{n}(a, p)\right) .
\end{aligned}
$$

Now (2.3) and (2.5) imply (2.2). 
Corollary 1. If $F$ is a strictly monotone convex function, then

$$
\begin{array}{r}
Q_{n}\left[A_{n}(b, q)-F\left(\frac{q_{n} P_{n}}{p_{n} Q_{n}} A_{n}\left(F^{-1}(b), p\right)\right)\right] \\
\geq Q_{n-1}\left[A_{n-1}(b, q)-F\left(\frac{q_{n} P_{n-1}}{p_{n} Q_{n-1}} A_{n-1}\left(F^{-1}(b), p\right)\right)\right] .
\end{array}
$$

Proof. It is enough to substitute $t=0$ and $a_{k}=F^{-1}\left(b_{k}\right), k=1,2, \ldots, n$ in (2.1).

Corollary 2. If $F$ is a convex, differentiable function, then for every $t \in \mathbb{R}$

$$
F\left(A_{n}(a, p)\right) \leq A_{n}\left(F\left(t+a_{k}\right), p\right)-t A_{n}\left(F^{\prime}\left(A_{k}(a, p), p\right) .\right.
$$

Proof. Substitute $(p)=(q)$ in (2.1) for $n=2,3, \ldots, n-1, n$ in order to get a sequence of inequalities. Then sum these inequalities.

Inequality (2.6) turns into the inequality of Redhefer upon taking $F(u)=e^{-u}$, $a_{i}=\ln \frac{1}{b_{i}}$ and $(p)=(1, \ldots, 1)$. This inequality is proved in [5] for $t \geq 0$. The same inequality is proved in [2], but only for $0 \leq t \leq 2$.

Theorem 4 ensures the statement of Theorem 2 proved in [2] only for $0 \leq t q_{n} \leq$ $Q_{n}$.

Corollary 3. For every $t \in \mathbb{R}$

$$
\begin{array}{r}
Q_{n}\left(A_{n}(b, q) e^{-t}+t \Gamma_{n}(b, q)-G_{n}(b, q)\right) \\
\geq Q_{n-1}\left(A_{n-1}(b, q) e^{-t}+t \Gamma_{n-1}(b, q)-G_{n-1}(b, q)\right) .
\end{array}
$$

Proof. To obtain (2.7), substitute $(p)=(q)$ and $F(u)=e^{-u}$ in (2.1). Then $F\left(a_{k}\right)=b_{k}=e^{-a_{k}}$ and $a_{k}=-\ln b_{k}=\ln \frac{1}{b_{k}}$.

Remark. Theorem 3, (the first theorem of [3]), follows from Theorem 4. Substitute the composition $G F$ instead of the convex function $F$ in (2.2) and use the Jensen inequality for the concave function $G$ :

$$
Q_{n} G\left(A_{n}\left(F\left(t+a_{k}\right), q\right)\right) \geq Q_{n-1} G\left(A_{n-1}\left(F\left(t+a_{k}\right), q\right)\right)+q_{n} G\left(F\left(t+a_{n}\right)\right) \text {. }
$$

The next inequality from [1] is an immediate consequence of Theorem 3.

Corollary 4. Suppose that $F$ is a strictly monotone function, $G$ is a concave function and $G F$ is a convex function. Then

$$
\begin{array}{r}
Q_{n}\left\{G\left(A_{n}(b, q)\right)-(G F)\left(\frac{q_{n} P_{n}}{p_{n} Q_{n}} A_{n}\left(F^{-1}(b), p\right)\right)\right\} \\
\geq Q_{n-1}\left\{G\left(A_{n-1}(b, q)\right)-(G F)\left(\frac{q_{n} P_{n-1}}{p_{n} Q_{n-1}} A_{n-1}\left(F^{-1}(b), p\right)\right)\right\} .
\end{array}
$$


Taking $(p)=(q)$, the next corollary is obtained from (2.1).

Corollary 5. If $F$ is a differentiable convex function, then

$$
\begin{array}{r}
\sum_{k=1}^{n} p_{k} F\left(t+a_{k}\right)-t \cdot \sum_{k=1}^{n} p_{k} F^{\prime}\left(\frac{1}{P_{k}} \sum_{j=1}^{k} p_{j} a_{j}\right) \\
-\sum_{k=1}^{n} p_{k} \cdot F\left(\frac{1}{P_{n}} \sum_{k=1}^{n} p_{k} a_{k}\right) \\
\geq \sum_{k=1}^{n-1} p_{k} F\left(t+a_{k}\right)-t \cdot \sum_{k=1}^{n-1} p_{k} F^{\prime}\left(\frac{1}{P_{k}} \sum_{j=1}^{k} p_{j} a_{j}\right) \\
-\sum_{k=1}^{n-1} p_{k} \cdot F\left(\frac{1}{P_{n-1}} \sum_{k=1}^{n-1} p_{k} a_{k}\right),
\end{array}
$$

whereby $t \in \mathbb{R}$.

\section{An Application on InTEgRal anAlogue}

Integral analogue is given in [3] as Theorem 2.

Theorem 5 (Godunova). Let $\psi$ be a convex, differentiable function and let $p(\tau)$ and $a(\tau)$ be positive functions for $\tau>0$. Then the function

$$
\begin{array}{r}
\Phi(x)=\int_{0}^{x} \psi(t+a(\tau)) p(\tau) d \tau-t \int_{0}^{x} \psi^{\prime}\left(\frac{\int_{0}^{\tau} a(\xi) p(\xi) d \xi}{\int_{0}^{\tau} p(\xi) d \xi}\right) p(\tau) d \tau \\
-\psi\left(\frac{\int_{0}^{x} a(\tau) p(\tau) d \tau}{\int_{0}^{x} p(\tau) d \tau}\right) \cdot \int_{0}^{x} p(\tau) d \tau
\end{array}
$$

is an increasing function on $(0, \infty)$.

The next corollary estimates the difference between the values, so that composition $G F$ is calculated at the mean values on the intervals $[0, x] \subset[0, y]$.

Corollary 6. Let $G F$ be a convex function, let $G$ be a concave function and suppose that $a, p$ are positive on $(0, \infty)$. Then

$$
\begin{array}{r}
G F\left(\frac{\int_{0}^{y} a(\tau) p(\tau) d \tau}{\int_{0}^{y} p(\tau) d \tau}\right) \cdot \int_{0}^{y} p(\tau) d \tau \\
-G F\left(\frac{\int_{0}^{x} a(\tau) p(\tau) d \tau}{\int_{0}^{x} p(\tau) d \tau}\right) \cdot \int_{0}^{x} p(\tau) d \tau
\end{array}
$$




$$
\begin{array}{r}
\leq \int_{x}^{y} p(\tau) d \tau \cdot G\left(\frac{\int_{x}^{y} F(t+a(\tau)) p(\tau) d \tau}{\int_{x}^{y} p(\tau) d \tau}\right) \\
-t \int_{x}^{y}(G F)^{\prime}\left(\frac{\int_{0}^{\tau} a(\xi) p(\xi) d \xi}{\int_{0}^{\tau} p(\xi) d \xi}\right) p(\tau) d \tau .
\end{array}
$$

Proof. Substitute $\psi=G F$ in (5). Notice that if

$$
\begin{aligned}
& \Phi(x)=\int_{0}^{x} G F(t+a(\tau)) p(\tau) d \tau+D(x), \text { then for } 0 \leq x \leq y \text { we have } \\
& D(x)-D(y) \\
&= t \int_{0}^{y}(G F)^{\prime}\left(\frac{\int_{0}^{\tau} a(\xi) p(\xi) d \xi}{\int_{0}^{\tau} p(\xi) d \xi}\right) p(\tau) d \tau \\
&+G F\left(\frac{\int_{0}^{y} a(\tau) p(\tau) d \tau}{\int_{0}^{y} p(\tau) d \tau}\right) \cdot \int_{0}^{y} p(\tau) d \tau \\
&-t \int_{0}^{x}(G F)^{\prime}\left(\frac{\int_{0}^{\tau} a(\xi) p(\xi) d \xi}{\int_{0}^{\tau} p(\xi) d \xi}\right) p(\tau) d \tau \\
&-G F\left(\frac{\int_{0}^{x} a(\tau) p(\tau) d \tau}{\int_{0}^{x} p(\tau) d \tau}\right) \cdot \int_{0}^{x} p(\tau) d \tau \\
& \leq \int_{0}^{y} G F(t+a(\tau)) p(\tau) d \tau-\int_{0}^{x} G F(t+a(\tau)) p(\tau) d \tau \\
&= \int_{x}^{y} G F(t+a(\tau)) p(\tau) d \tau \\
& \leq \int_{x}^{y} p(\tau) d \tau \cdot G\left(\frac{\int_{x}^{y} F(t+a(\tau)) p(\tau) d \tau}{\int_{x}^{y} p(\tau) d \tau}\right) .
\end{aligned}
$$

In case that all calculations are well defined, Jensen's inequality for a concave function $G$

$$
\int_{0}^{y} G F(t+a(\tau)) p(\tau) d \tau \leq P(y) \cdot G\left(\frac{1}{P(y)} \int_{0}^{y} F(t+a(\tau)) p(\tau) d \tau\right),
$$

with $P(y)=\int_{0}^{y} p(\tau) d \tau$, makes it possible to define the function

$$
\mathscr{F}(y)=P(y) \cdot G\left(\frac{1}{P(y)} \int_{0}^{y} F(t+a(\tau)) p(\tau) d \tau\right)-\int_{0}^{y} G F(t+a(\tau)) p(\tau) d \tau .
$$

The function $\mathcal{F}$ is monotone on $(0, \infty)$. 
Proposition 1. If $G$ is a concave function, then $0 \leq x \leq y$ ensures

$$
\mathcal{F}(x) \leq \mathcal{F}(y) .
$$

Proof. This statements follows from the concavity of $G$. There is a chain of inequalities:

$$
\begin{array}{r}
P(x) G\left(\frac{1}{P(x)} \int_{0}^{x} F(t+a(\tau)) p(\tau) d \tau\right)-\int_{0}^{x} G F(t+a(\tau)) p(\tau) d \tau \\
+\int_{0}^{y} G F(t+a(\tau)) p(\tau) d \tau \\
=P(x) G\left(\frac{1}{P(x)} \int_{0}^{x} F(t+a(\tau)) p(\tau) d \tau\right) \\
+\frac{\int_{x}^{y} p(\tau) d \tau}{\int_{x}^{y} p(\tau) d \tau} \cdot \int_{x}^{y} G F(t+a(\tau)) p(\tau) d \tau \\
\leq \frac{P(y)}{P(y)}\left[P(x) \cdot G\left(\frac{1}{P(x)} \int_{0}^{x} F(t+a(\tau)) p(\tau) d \tau\right)\right. \\
\left.+\int_{x}^{y} p(\tau) d \tau \cdot G\left(\frac{\left.\int_{x}^{y} F(t+a(\tau)) p(\tau) d \tau\right)}{\int_{x}^{y} p(\tau) d \tau}\right)\right] \\
=P(y)\left[\frac{P(x)}{P(y)} \cdot G\left(\frac{1}{P(x)} \int_{0}^{x} F(t+a(\tau)) p(\tau) d \tau\right)\right. \\
\left.+\frac{\int_{x}^{y} p(\tau) d \tau}{P(y)} \cdot G\left(\frac{\left.\int_{x}^{y} F(t+a(\tau)) p(\tau) d \tau\right)}{\int_{x}^{y} p(\tau) d \tau}\right)\right] \\
\leq P(y) \cdot G\left(\frac{1}{P(y)} \int_{0}^{x} F(t+a(\tau)) p(\tau) d \tau+\frac{1}{P(y)} \int_{x}^{y} F(t+a(\tau)) p(\tau) d \tau\right) \\
=P(y) \cdot G\left(\frac{1}{P(y)} \int_{0}^{y} F(t+a(\tau)) p(\tau) d \tau\right) .
\end{array}
$$

At this point observe certain refinements when $G$ is concave and $G F$ is a convex function.

Remark 1. Applying (3.2) in

$$
D(x)-D(y) \leq \int_{0}^{y} G F(t+a(\tau)) p(\tau) d \tau-\int_{0}^{x} G F(t+a(\tau)) p(\tau) d \tau,
$$

the following is obtained:

$$
P(y) \cdot G F\left(\frac{\int_{0}^{y} a(\tau) p(\tau) d \tau}{\int_{0}^{y} p(\tau) d \tau}\right)-P(x) \cdot G F\left(\frac{\int_{0}^{x} a(\tau) p(\tau) d \tau}{\int_{0}^{x} p(\tau) d \tau}\right)
$$




$$
\begin{array}{r}
\leq P(y) \cdot G\left(\frac{\int_{0}^{y} F(t+a(\tau)) p(\tau) d \tau}{\int_{0}^{y} p(\tau) d \tau}\right)-P(x) \cdot G\left(\frac{\int_{0}^{x} F(t+a(\tau)) p(\tau) d \tau}{\int_{0}^{x} p(\tau) d \tau}\right) \\
-t \int_{x}^{y}(G F)^{\prime}\left(\frac{\int_{0}^{\tau} a(\tau) p(\tau) d \tau}{\int_{0}^{y} p(\tau) d \tau}\right) p(\tau) d \tau .
\end{array}
$$

To sum up, if $G F$ is a non-decreasing function, we can make the following observation.

Remark 2. If $G F$ is a nondecreasing differentiable function, then $G F^{\prime} \geq 0$ and for $t \geq 0$ one can obtain

$$
\begin{array}{r}
P(y) \cdot G F\left(\frac{\int_{0}^{y} a(\tau) p(\tau) d \tau}{\int_{0}^{y} p(\tau) d \tau}\right)-P(x) \cdot G F\left(\frac{\int_{0}^{x} a(\tau) p(\tau) d \tau}{\int_{0}^{x} p(\tau) d \tau}\right) \\
\leq P(y) \cdot G\left(\frac{\int_{0}^{y} F(t+a(\tau)) p(\tau) d \tau}{\int_{0}^{y} p(\tau) d \tau}\right)-P(x) \cdot G\left(\frac{\int_{0}^{x} F(t+a(\tau)) p(\tau) d \tau}{\int_{0}^{x} p(\tau) d \tau}\right) .
\end{array}
$$

For $t=0$ we get ordinary monotonicity. If $[0, x] \subseteq[0, y]$, then

$$
\begin{aligned}
& P(x) \cdot G\left(\frac{\int_{0}^{x} F(a(\tau)) p(\tau) d \tau}{\int_{0}^{x} p(\tau) d \tau}\right)-P(x) \cdot G F\left(\frac{\int_{0}^{x} a(\tau) p(\tau) d \tau}{\int_{0}^{x} p(\tau) d \tau}\right) \\
\leq & P(y) \cdot G\left(\frac{\int_{0}^{y} F(a(\tau)) p(\tau) d \tau}{\int_{0}^{y} p(\tau) d \tau}\right)-P(y) \cdot G F\left(\frac{\int_{0}^{y} a(\tau) p(\tau) d \tau}{\int_{0}^{y} p(\tau) d \tau}\right) .
\end{aligned}
$$

\section{DECREASING PROPERTY UPON TAKING SUBSETS}

In the next theorem an integral version of the statement obtained in (1.6) is given whereby $(p)=(q)$ and $t=0$. The statement appeared as particular case of Bullen's inequality (see [4]). In [3] the author omitted the proof. We are providing it here to refresh certain basic integral inequalities.

Theorem 6. Let $\mathscr{D}$ be a measurable domain and let $\mathscr{D}_{1} \subset \mathcal{D}$ be its regular measurable sub-domain. Further, suppose $a, q: \mathscr{D} \rightarrow[0,+\infty)$ is such that $Q=$ $\int_{\mathscr{D}} q(u) d V_{u} \neq 0$ and $Q_{1}=\int_{D_{1}} q(u) d V_{u} \neq 0$. If $G$ is concave, $F$ is monotone and $G F$ is a convex function, then

$$
\begin{gathered}
Q_{1}\left[G\left(\frac{1}{Q_{1}} \int_{D_{1}} F(a(u)) q(u) d V_{u}\right)-G F\left(\frac{1}{Q_{1}} \int_{D_{1}} a(u) q(u) d V_{u}\right)\right] \\
\leq Q\left[G\left(\frac{1}{Q} \int_{\mathscr{D}} F(a(u)) q(u) d V_{u}\right)-G F\left(\frac{1}{Q} \int_{\mathscr{D}} a(u) q(u) d V_{u}\right)\right] .
\end{gathered}
$$


Proof. Concavity of $G$ ensures

$$
\begin{aligned}
& Q \cdot G\left(\frac{1}{Q} \int_{\mathscr{D}} F(a(u)) q(u) d V_{u}\right) \\
\geq & Q_{1} \cdot G\left(\frac{1}{Q_{1}} \int_{D_{1}} F(a(u)) q(u) d V_{u}\right) \\
& +Q_{2} \cdot G\left(\frac{1}{Q_{2}} \int_{D_{2}} F(a(u)) q(u) d V_{u}\right),
\end{aligned}
$$

wherby $\mathscr{D}_{2}=\mathscr{D} \backslash \mathscr{D}_{1}$ with $Q_{2}=\int_{\mathscr{D}_{2}} q(u) d V_{u} \neq 0$. Besides, the convexity of $G F$ implies

$$
\begin{aligned}
& Q \cdot G F\left(\frac{1}{Q} \int_{\mathscr{D}} a(u) q(u) d V_{u}\right) \\
& \leq Q_{1} \cdot G F\left(\frac{1}{Q_{1}} \int_{D_{1}} a(u) q(u) d V_{u}\right)+Q_{2} \cdot G F\left(\frac{1}{Q_{2}} \int_{D_{2}} a(u) q(u) d V_{u}\right) .
\end{aligned}
$$

Integral version of Jensen's inequality (see [4], p. 45) for concave function $G$ provides

$$
Q_{2} \cdot G\left(\frac{1}{Q_{2}} \int_{D_{2}} F(a(u)) q(u) d V_{u}\right) \geq \int_{D_{2}} G F(a(u)) q(u) d V_{u} .
$$

Furthermore, the convexity of $G F$ provides the statement

$$
\int_{D_{2}} G F(a(u)) q(u) d V_{u} \geq Q_{2} \cdot G F\left(\frac{1}{Q_{2}} \int_{D_{2}} a(u) q(u) d V_{u}\right) .
$$

Now there is a chain

$$
\begin{aligned}
& Q \cdot G\left(\frac{1}{Q} \int_{\mathscr{D}} F(a(u)) q(u) d V_{u}\right)-Q_{1} \cdot G\left(\frac{1}{Q_{1}} \int_{D_{1}} F(a(u)) q(u) d V_{u}\right) \\
= & Q_{2} \cdot G\left(\frac{1}{Q_{2}} \int_{D_{2}} F(a(u)) q(u) d V_{u}\right) \geq Q_{2} \cdot G F\left(\frac{1}{Q_{2}} \int_{D_{2}} a(u) q(u) d V_{u}\right) \\
\geq & Q \cdot G F\left(\frac{1}{Q} \int_{\mathscr{D}} a(u) q(u) d V_{u}\right)-Q_{1} \cdot G F\left(\frac{1}{Q_{1}} \int_{D_{1}} a(u) q(u) d V_{u}\right) .
\end{aligned}
$$

The first line and the last line from this give (4.1).

Finally, we generalize (4.1).

Theorem 7. Let $(\Omega, \Sigma, \mu)$ be a space with a positive finite measure and let a : $\Omega \rightarrow \mathbb{R}$ be a $\mu$-measurable function. Suppose that $G$ and $F$ are differentiable functions such that $G$ is concave and $G F$ is convex. For every $t \in \mathbb{R}$, if $\Omega_{1} \in \Sigma$, and $\Omega_{1} \neq \Omega$, then

$$
\mu(\Omega) \cdot G\left(\frac{1}{\mu(\Omega)} \int_{\Omega} F(t+a) d \mu\right)
$$




$$
\begin{array}{r}
-\mu\left(\Omega_{1}\right) \cdot G\left(\frac{1}{\mu\left(\Omega_{1}\right)} \int_{\Omega_{1}} F(t+a) d \mu\right) \\
\geq\left(\mu(\Omega)-\mu\left(\Omega_{1}\right)\left[G F\left(\frac{\int_{\Omega} a d \mu-\int_{\Omega_{1}} a d \mu}{\mu(\Omega)-\mu\left(\Omega_{1}\right)}\right)\right.\right. \\
\left.+t \cdot(G F)^{\prime}\left(\frac{\int_{\Omega} a d \mu-\int_{\Omega_{1}} a d \mu}{\mu(\Omega)-\mu\left(\Omega_{1}\right)}\right)\right] .
\end{array}
$$

Proof. Take $\Omega \backslash \Omega_{1}=\Omega_{2}$. Then concavity of $G$ ensures

$$
\begin{aligned}
\mu(\Omega) \cdot G\left(\frac{1}{\mu(\Omega)} \int_{\Omega} F(t+a) d \mu\right) & -\mu\left(\Omega_{1}\right) \cdot G\left(\frac{1}{\mu\left(\Omega_{1}\right)} \int_{\Omega_{1}} F(t+a) d \mu\right) \\
& \geq \mu\left(\Omega_{2}\right) \cdot G\left(\frac{1}{\mu\left(\Omega_{2}\right)} \int_{\Omega_{2}} F(t+a) d \mu\right) .
\end{aligned}
$$

Concavity of $G$ implies (see [4], p.51) that

$$
\mu\left(\Omega_{2}\right) \cdot G\left(\frac{1}{\mu\left(\Omega_{2}\right)} \int_{\Omega_{2}} F(t+a) d \mu\right) \geq \int_{\Omega_{2}} G F(t+a) d \mu .
$$

Now, convexity of $G F$ implies

$$
\int_{\Omega_{2}} G F(t+a) d \mu \geq \mu\left(\Omega_{2}\right) \cdot G F\left(\frac{1}{\mu\left(\Omega_{2}\right)} \int_{\Omega_{2}}(t+a) d \mu\right) .
$$

Using (2.4) and the equality $\frac{1}{\mu\left(\Omega_{2}\right)} \int_{\Omega_{2}}(t+a) d \mu=t+\frac{1}{\mu\left(\Omega_{2}\right)} \int_{\Omega_{2}} a d \mu$, we claim that

$$
\begin{array}{r}
G F\left(t+\frac{1}{\mu\left(\Omega_{2}\right)} \int_{\Omega_{2}} a d \mu\right) \geq G F\left(\frac{1}{\mu\left(\Omega_{2}\right)} \int_{\Omega_{2}} a d \mu\right) \\
+t(G F)^{\prime}\left(\frac{1}{\mu\left(\Omega_{2}\right)} \int_{\Omega_{2}} a d \mu\right) .
\end{array}
$$

Finally, (4.3) and (4.4), together with the well-known properties $\mu\left(\Omega \backslash \Omega_{1}\right)=\mu(\Omega)-\mu\left(\Omega_{1}\right)$ and $\int_{\Omega \backslash \Omega_{1}} a d \mu=\int_{\Omega} a d \mu-\int_{\Omega_{1}} a d \mu$ give (4.2).

Theorem 7 contains (4.1) as a particular case.

Remark 3. Notice that convexity of $G F$, assumed in (4.4), implies

$$
\begin{array}{r}
\mu(\Omega) \cdot G\left(\frac{1}{\mu(\Omega)} \int_{\Omega} F(t+a) d \mu\right)-\mu\left(\Omega_{1}\right) \cdot G\left(\frac{1}{\mu\left(\Omega_{1}\right)} \int_{\Omega_{1}} F(t+a) d \mu\right) \\
\geq \mu(\Omega) \cdot G F\left(\frac{1}{\mu(\Omega)} \int_{\Omega} a d \mu\right)-\mu\left(\Omega_{1}\right) \cdot G F\left(\frac{1}{\mu\left(\Omega_{1}\right)} \int_{\Omega_{1}} a d \mu\right)
\end{array}
$$




$$
+t \cdot(G F)^{\prime}\left(\frac{\int_{\Omega} a d \mu-\int_{\Omega_{1}} a d \mu}{\mu(\Omega)-\mu\left(\Omega_{1}\right)}\right)
$$

and (4.1) can be obtained from (4.5) for $t=0$.

In the similar manner as in [6], where the authors have refined Jensen's inequality, here we give an estimate after dividing the $n$-tuples $\left(a_{1}, \ldots, a_{n}\right),\left(p_{1}, \ldots, p_{n}\right)$ and $\left(q_{1}, \ldots, q_{n}\right)$ into groups. Firstly, we obtain the next Theorem.

Theorem 8. Suppose that $G$ and $F$ are differentiable functions such that $G$ is concave and $G F$ is convex. If $m \leq n$, then

$$
\begin{array}{r}
Q_{n}\left[G\left(A_{n}\left(F\left(t+a_{k}\right), q\right)\right)-t A_{n}\left((G F)^{\prime}\left(\frac{q_{k} P_{k}}{p_{k} Q_{k}} A_{k}(a, p)\right), q\right)\right. \\
\left.-G F\left(\frac{q_{n} P_{n}}{p_{n} Q_{n}} A_{n}(a, p)\right)\right] \\
-Q_{m}\left[G\left(A_{m}\left(F\left(t+a_{k}\right), q\right)\right)-t A_{m}\left((G F)^{\prime}\left(\frac{q_{k} P_{k}}{p_{k} Q_{k}} A_{k}(a, p)\right), q\right)\right. \\
\left.-G F\left(\frac{q_{m} P_{m}}{p_{m} Q_{m}} A_{m}(a, p)\right)\right] \\
\geq \sum_{k=m}^{n-1} Q_{k}\left[G F\left(\frac{q_{k} P_{k}}{p_{k} Q_{k}} A_{k}(a, p)\right)-\left(\frac{q_{k+1} P_{k}}{p_{k+1} Q_{k}} A_{k}(a, p)\right)\right] .
\end{array}
$$

Proof. No generality is lost if we separate each of $n$-tuples $\left(a_{1}, \ldots, a_{n}\right),\left(p_{1}, \ldots, p_{n}\right)$ and $\left(q_{1}, \ldots, q_{n}\right)$ by the choosing the first $m$ members in the first group. For example: $\left(a_{1}, \ldots, a_{m}, a_{m+1}, \ldots, a_{n}\right)$.

Then the first inequality is

$$
\begin{array}{r}
Q_{n}\left[G\left(A_{n}\left(F\left(t+a_{k}\right), q\right)\right)-t A_{n}\left((G F)^{\prime}\left(\frac{q_{k} P_{k}}{p_{k} Q_{k}} A_{k}(a, p)\right), q\right)\right. \\
\left.-G F\left(\frac{q_{n} P_{n}}{p_{n} Q_{n}} A_{n}(a, p)\right)\right] \\
\geq Q_{n-1}\left[G\left(A_{n-1}\left(F\left(t+a_{k}\right), q\right)\right)-t A_{n-1}\left((G F)^{\prime}\left(\frac{q_{k} P_{k}}{p_{k} Q_{k}} A_{k}(a, p)\right), q\right)\right. \\
\left.-G F\left(\frac{q_{n-1} P_{n-1}}{p_{n-1} Q_{n-1}} A_{n-1}(a, p)\right)\right] \\
+Q_{n-1}\left[G F\left(\frac{q_{n-1} P_{n-1}}{p_{n-1} Q_{n-1}} A_{n-1}(a, p)\right)-G F\left(\frac{q_{n} P_{n-1}}{p_{n} Q_{n-1}} A_{n-1}(a, p)\right)\right] .
\end{array}
$$

The second one is

$$
Q_{n-1}\left[G\left(A_{n-1}\left(F\left(t+a_{k}\right), q\right)\right)-t A_{n-1}\left((G F)^{\prime}\left(\frac{q_{k} P_{k}}{p_{k} Q_{k}} A_{k}(a, p)\right), q\right)\right.
$$




$$
\begin{aligned}
& \left.-G F\left(\frac{q_{n-1} P_{n-1}}{p_{n-1} Q_{n-1}} A_{n-1}(a, p)\right)\right] \\
& \geq Q_{n-2}\left[G\left(A_{n-2}\left(F\left(t+a_{k}\right), q\right)\right)-t A_{n-2}\left((G F)^{\prime}\left(\frac{q_{k} P_{k}}{p_{k} Q_{k}} A_{k}(a, p)\right), q\right)\right. \\
& \left.-G F\left(\frac{q_{n-2} P_{n-2}}{p_{n-2} Q_{n-2}} A_{n-2}(a, p)\right)\right] \\
& +Q_{n-2}\left[G F\left(\frac{q_{n-2} P_{n-2}}{p_{n-2} Q_{n-2}} A_{n-2}(a, p)\right)-G F \% \text { left }\left(\frac{q_{n-1} P_{n-2}}{p_{n-1} Q_{n-2}} A_{n-2}(a, p)\right)\right.
\end{aligned}
$$

And finally, the last one is

$$
\begin{array}{r}
Q_{m+1}\left[G\left(A_{m+1}\left(F\left(t+a_{k}\right), q\right)\right)-t A_{m+1}\left((G F)^{\prime}\left(\frac{q_{k} P_{k}}{p_{k} Q_{k}} A_{k}(a, p)\right), q\right)\right. \\
\left.-G F\left(\frac{q_{m+1} P_{m+1}}{p_{m+1} Q_{m+1}} A_{m+1}(a, p)\right)\right] \\
\geq Q_{m}\left[G\left(A_{m}\left(F\left(t+a_{k}\right), q\right)\right)-t A_{m}\left((G F)^{\prime}\left(\frac{q_{k} P_{k}}{p_{k} Q_{k}} A_{k}(a, p)\right), q\right)\right] . \\
\left.-G F\left(\frac{q_{m} P_{m}}{p_{m} Q_{m}} A_{m}(a, p)\right)\right] \\
+Q_{m}\left[G F\left(\frac{q_{m} P_{m}}{p_{m} Q_{m}} A_{m}(a, p)\right)-G F\left(\frac{q_{m+1} P_{m}}{p_{m+1} Q_{m}} A_{m}(a, p)\right)\right] .
\end{array}
$$

The desired inequality in the statement is obtained by summing these inequalities.

The sum $\sum_{k=m}^{n-1} Q_{k}\left[G F\left(\frac{q_{k} P_{k}}{p_{k} Q_{k}} A_{k}(a, p)\right)-\left(\frac{q_{k+1} P_{k}}{p_{k+1} Q_{k}} A_{k}(a, p)\right)\right]$ depends on the positive weights $(p)$ and $(q)$. Therefore it could be positive, negative or zero. When $(p)=(q)$, the sum is zero and and we obtain the following Corollary.

Corollary 7. Suppose $G$ and $F$ fulfill the assumptions of Theorem 8. If $n \geq m$, then

$$
\begin{array}{r}
Q_{n}\left[G\left(A_{n}\left(F\left(t+a_{k}\right), p\right)\right)-t A_{n}\left((G F)^{\prime}\left(A_{k}(a, p)\right), p\right)-G F\left(A_{n}(a, p)\right)\right] \\
\geq Q_{m}\left[G\left(A_{m}\left(F\left(t+a_{k}\right), p\right)\right)-t A_{m}\left((G F)^{\prime}\left(A_{k}(a, p)\right), p\right)-G F\left(A_{m}(a, p)\right)\right] .
\end{array}
$$

Finally, we state the following Corollary in the case of disjunct separation of the $n$-tuples as: $\left(a_{1}, \ldots, a_{n}\right)=\left(a_{1}, \ldots, a_{m}, a_{m+1}, \ldots, a_{n}\right)$.

Corollary 8. Suppose that $G(u)$ and $F(u)$ satisfy conditions given in Theorem 8. If $l+m=n$ and $\left(b_{1}, \ldots, b_{l}, c_{1}, \ldots, c_{m}\right)=\left(a_{1}, \ldots, a_{n}\right)$, then

$$
Q_{l}\left\{G\left(A_{l}\left(F\left(t+b_{k}\right), p\right)\right)-t A_{l}\left((G F)^{\prime}\left(A_{k}(b, p)\right), p\right)\right.
$$




$$
\begin{array}{r}
\left.-G F\left(A_{l}(b, p)\right)\right\} \\
+Q_{m}\left\{G\left(A_{m}\left(F\left(t+c_{k}\right), q\right)\right)-t A_{m}\left((G F)^{\prime}\left(A_{k}(c, p)\right), p\right)\right. \\
\left.-G F\left(A_{m}(c, p)\right)\right\} \\
\leq 2 \cdot Q_{n}\left\{G\left(A_{n}\left(F\left(t+a_{k}\right), p\right)\right)-t A_{n}\left((G F)^{\prime}\left(A_{k}(a, p)\right), p\right)\right. \\
\left.-G F\left(A_{n}(a, p)\right)\right\} .
\end{array}
$$

\section{REFERENCES}

[1] P. S. Bullen, "An inequality due to Henrici," Univ. Beograd. Publ. Elektrotehn. Fak. Ser. Mat. Fiz., no. 247-273, pp. 21-26, 1969.

[2] P. S. Bullen, "An inequality of Redheffer," Univ. Beograd. Publ. Elektrotehn. Fak. Ser. Mat. Fiz., no. 274-301, pp. 25-26, 1969.

[3] E. K. Godunova, "Generalisation and integral analogues of some Bullen's inequality," manuscript, 1970.

[4] J. E. Pečarič, F. Proschan, and Y. L. Tong, Convex functions, partial orderings, and statistical applications, ser. Mathematics in Science and Engineering. Boston, MA: Academic Press, Inc., 1992, vol. 187.

[5] R. Redheffer, "Recurrent inequalities," Proc. London Math. Soc. (3), vol. 17, pp. 683-699, 1967.

[6] V. Čuljak and B. Ivanković, "One refinement of Jensen's discrete inequality and applications," Math. Inequal. Appl., vol. 12, no. 4, pp. 805-814, 2009.

\section{Authors' addresses}

\section{Božidar Ivanković}

Faculty of Transport and Trafic Engineering, University of Zagreb, Vukelićeva 4, 10000, Croatia

E-mail address: bozidar.ivankovicezg.t-com.hr

\section{Josip Pečarić}

Faculty of Textile, University of Zagreb, Prilaz Baruna Filipovića 30, 10000 Zagreb, Croatia

E-mail address: pecaricdelement.hr

\section{Sanja Varošanec}

Department of Mathematics, University of Zagreb, Croatia

E-mail address: varosans@math.hr 